2019-08-06

\title{
Wetting Metamorphosis of Hydrophobic Fluoropolymer Coatings Submerged in Water and Ultrasonically Vibrated
}

\author{
Matthew Trapuzzano \\ University of South Florida \\ Nathan B. Crane \\ Brigham Young University - Provo, nbcrane@byu.edu \\ Rasim Guldiken \\ University of South Florida \\ Andrés Tejada-Martínez \\ University of South Florida \\ Follow this and additional works at: https://scholarsarchive.byu.edu/facpub \\ Part of the Manufacturing Commons, and the Other Mechanical Engineering Commons
}

\section{Original Publication Citation \\ Matthew Trapuzzano, Nathan B. Crane, Rasim Guldiken, and Andres Tejada-Martinez, “Wetting Metamorphosis of Hydrophobic Fluoropolymer Coatings Submerged in Water and Ultrasonically Vibrated," Journal of Coatings Technology Research (JCTR), Published 8/6/2019.}

\section{BYU ScholarsArchive Citation}

Trapuzzano, Matthew; Crane, Nathan B.; Guldiken, Rasim; and Tejada-Martínez, Andrés, "Wetting Metamorphosis of Hydrophobic Fluoropolymer Coatings Submerged in Water and Ultrasonically Vibrated" (2019). Faculty Publications. 5343.

https://scholarsarchive.byu.edu/facpub/5343

This Peer-Reviewed Article is brought to you for free and open access by BYU ScholarsArchive. It has been accepted for inclusion in Faculty Publications by an authorized administrator of BYU ScholarsArchive. For more information, please contact ellen_amatangelo@byu.edu. 


\title{
WETTING METAMORPHOSIS OF HYDROPHOBIC FLUOROPOLYMER COATINGS SUBMERGED IN WATER AND ULTRASONICALLY VIBRATED
}

\author{
Primary author for correspondence: \\ Matthew Trapuzzano: Researcher, University of South Florida \\ Email: mtrapuzz@mail.usf.edu, Phone: 412-965-8897 \\ Secondary authors: \\ Nathan B. Crane, Ph.D. Brigham Young University \\ Email: nbcrane@,byu.edu \\ Rasim Guldiken, Ph.D. University of South Florida \\ Email: guldiken@usf.edu \\ Andrés Tejada-Martínez, Ph.D. University of South Florida \\ Email: aetejada@usf.edu
}

\begin{abstract}
Many important processes, from manufacture of integrated circuit boards, to an insect's ability to walk on water, depend on the wetting of liquids on surfaces. Wetting is commonly controlled through material selection, coatings, and/or surface texture. However, wetting is sensitive to environmental conditions. In particular, some hydrophobic fluoropolymer coatings are sensitive to extended water exposure as evidenced by a declining contact angle and increasing contact angle hysteresis. Understanding "degradation" of these coatings is critical to applications that employ them. The durability of a series of fluoropolymer coatings were tested by measuring the contact angle before, during, and after extended submersion of coated glass slides in deionized water. These measurements were compared to similar measurements taken before, during, and after the same coatings were subject to ultrasonic vibration while covered in deionized water. Both methods caused changes in advancing and receding contact angles, but degradation rates of vibrated coatings were significantly increased. Prolonged soaking caused significant decreases in the contact angle of most of the samples, though most experienced significant recovery of hydrophobicity when heat-treated at $160^{\circ} \mathrm{C}$ after submersion. Some coatings appear noticeably more resistant to degradation by one or both methods. FluoroSyl showed no clear change under submersion, while other coatings experienced significant contact angle change. Degradation of vibrated coatings is inconsistent among coatings, and is not simply an acceleration of the degradation resulting from submerging coatings in water. This is apparent as some coatings are affected by one method but not the other. Atomic force microscopy revealed differing surface effects for different coatings after the coatings were submerged and heat-treated, but the magnitude of this roughness change is insufficient to fully explain the wetting changes. The rate of contact angle degradation was not altered significantly for coatings that were vibrated with acceleration magnitudes between 700 and $7000 \mathrm{~g}$.
\end{abstract}




\section{KEYWORDS}

Fluoropolymer; Ultrasonic; Hydrophobic; Contact Angle Hysteresis; Degradation

\section{INTRODUCTION}

Wetting is commonly controlled through material selection, coatings, and/or surface texture, however these means are sensitive to environmental conditions (heat, light, moisture, abrasion), especially in monolayer coatings. Much scientific literature exists on the degradation of coatings, including effects of UV exposure, ${ }^{1,2}$ temperature, ${ }^{3-5}$ exposure to water, ${ }^{6-9}$ or mechanical erosion. ${ }^{10}$ The effects of various means of degradation varies from coating to coating, suggesting that coating composition plays a major role in the robustness of the coating.

Hydrophobic, or water-repellant coatings are one type of coating that has become increasingly common in every-day applications. Fluoropolymer, coatings, especially polytetrofluoroethelyne (PTFE), are perhaps the most common types of hydrophobic coatings. The low surface energy of fluoropolymer coatings causes liquids wetting the surface to "bead" up and easily roll off of the surface. Hydrophobic coatings can be found in nature, ${ }^{11}$ and have been used for the prevention of biofouling ${ }^{12}$ and corrosion, ${ }^{13}$ as well as encapsulants for electronic components to improve the reliability when such components are exposed to moisture while in operation. ${ }^{14}{ }^{15}$ Use of hydrophobic coatings, specifically PTFE and a proprietary fluoropolymer coating called Cytop (Cytop CTX-809A, Bellex Corp.) are common in research and industry applications like electrowetting ${ }^{16}$ due to their low friction characteristics. These coatings are usually easy to apply and relatively inexpensive.

While hydrophobic coatings have many applications, reliability of the coatings is crucial to repeatable long-term wetting. Therefore, changes in contact angle (CA) and/or contact angle hysteresis $(\mathrm{CAH})$ of the coating is of great importance. Degradation of fluoropolymer coatings like FluoroPel, Cytop, and Teflon due to electrowetting actuation has been documented. ${ }^{17}$ Some hydrophobic fluoropolymer coatings are also sensitive to extended water exposure as evidenced by an increase in coating thickness or weight. ${ }^{18}$ This could be a cause for concern if water uptake is found to have an effect on the CA of these coatings. Cytop is reported to have a minimal water absorptivity at less than 0.01 percent at $60^{\circ} \mathrm{C}$ in water, ${ }^{19}$ so it may seem like a good candidate coating for applications where prolonged exposure to water occurs. However, this type of data is not readily available for many coatings, so information about how water exposure affects the CA of hydrophobic fluoropolymer coatings could be useful to determine which coatings would be appropriate for specific applications.

Sonication is a common method used for a variety of cleaning and preparatory processes due to the generation of high frequency pressure waves that create oscillating gas bubbles that implode, dislodging contaminants from a surface. Sonication has also been shown to increase mass transport in and out of permeable solids. ${ }^{20}$ If degradation of CA for hydrophobic fluoropolymer coatings can be attributed to uptake of water, sonication of these coatings under water exposure could accelerate this process by increasing transport at the coating/liquid interface. If so, this method could provide quick insight on coating performance under prolonged exposure to water.

This work uses standard CA and surface energy measurement methods to characterize the degradation of hydrophobic fluoropolymer coatings due to long term water exposure over the course of many days, and contrasts these with measurements from short term exposure under high frequency vibration $(>20 \mathrm{kHz}$ ) over a period of minutes. The goal is to quantify the wetting effects of coatings exposed to water for long periods of time, understand the causes, and determine if 
ultrasonic vibration provides an expedited method to predict the effects of long term water exposure for a given coating.

\section{EXPERIMENTAL METHODS}

Glass microscope slides (1 $\mathrm{mm}$ thick) were coated with several different commercial hydrophobic fluoropolymer coatings (Table 1). All coatings were purchased from manufacturers and are proprietary, so the exact constituents of each coating is unknown. Coatings listed in this table without an application method indicate that the slides were purchased with a pre-applied coating, while other coatings were applied to glass slides using the manufacturer recommended application methods. All glass slides were solvent-cleaned with acetone and then methanol, followed by rinsing with deionized water and then isopropyl alcohol before drying with $\mathrm{N}_{2}$. The glass slides were then plasma-cleaned for 2 minutes with an $\mathrm{O}_{2}$ plasma.

Table 1: Hydrophobic coatings used for testing, their application method, and their manufacturer.

\begin{tabular}{|c|c|c|}
\hline Coating & Application & Manufacturer \\
\hline Cytop CTX-809A & Spin & Bellex \\
\hline FluoroPel 1601V & Spin & Cytonix \\
\hline FluoroSyl 3750 & Dip & Cytonix \\
\hline FluorAcryl 3298 & Dip & Cytonix \\
\hline PTFE & N/A & EMS \\
\hline AFC 2101 & N/A & AFT Fluorotec \\
\hline AFC 2206 & N/A & AFT Fluorotec \\
\hline
\end{tabular}

Cytop is an amorphous fluoropolymer coating that can be applied by spin-coating, dip-coating, or by potting. ${ }^{21}$ Spin-coating was done at $2000 \mathrm{rpm}$ for 40 seconds. A soft-bake at $100^{\circ} \mathrm{C}$ on a hot plate for 90 seconds, and a hard-bake at $180^{\circ} \mathrm{C}$ for 30 minutes followed, resulting in a coating of $\sim 1 \mu \mathrm{m}$ thickness. ${ }^{21}$ FluoroPel is a polymer in a fluorosolvent. The recommended application method is spin-coating. ${ }^{22}$ For deposition, a drop of FluoroPel was placed in the center of the glass slide before spinning at $2000 \mathrm{rpm}$ for 40 seconds. A soft-bake at $100^{\circ} \mathrm{C}$ on a hot plate for 90 seconds, and a hard-bake at $150^{\circ} \mathrm{C}$ for 30 minutes followed. FluorAcryl is a fluoroacrylate UVcurable oligomer that can be applied by dip-coating, or spray-coating. ${ }^{23}$ Slides were coated by a manual dipping method. Once the coating settled flat, it was cured with $420 \mathrm{~nm}$ UV light to a pencil hardness greater than \#2. FluoroSyl is a perfluoropolyether coating that can be applied by dip-coating, or spray-coating. ${ }^{24}$ Slides were submerged into the FluoroSyl liquid for 1 minute. After 1 minute, glass slides were removed and hard-baked at $100^{\circ} \mathrm{C}$ for 20 minutes. Coatings that came pre-applied on glass slides were already prepared for testing, and were simply diced to manageable sizes.

Samples of all coated glass slides were tested for degradation by two methods (Figure 1). The first degradation method was continuous submersion of hydrophobic coatings in water. Coated glass slides were covered with deionized water and sealed in glass containers for a period of 130 days (Figure 1 (2a)). A total of 6 slides $(18.25 \mathrm{~mm} \times 18.25 \mathrm{~mm}$ ) of each coating were used for submersion testing. Each coating type was submerged in a separate container to prevent crosscontamination. Samples were removed at intervals for characterization, where advancing and receding CA measurments were taken at 2 arbitrary locations on the surface of each sample, resulting in a total of twelve measurements for each coating at each point of characterization. The coatings were then re-submerged until they were removed again for characterization. After 130 
days of submersion, one submerged sample of each coating was removed from the water bath, dried with compressed air, and heat-treated in an oven at $160^{\circ} \mathrm{C}$ for up to 20 days. Samples were again removed from the oven at intervals to measure advancing and receding CA at 4 arbitrary locations on the surface of each sample each time characterization occurred.

The second degradation method was vibration-accelerated degradation. This was tested by bonding samples of each coating on a glass slide to a piezoelectric transducer (PZT) actuation stack (Figure $1(2 \mathrm{~b})$ ) with adhesive (Loctite 4310). The coated glass slide was covered with a puddle of deionized water, and then vibrated orthogonally to the coated surface (Figure 1(3b)) at a frequency of $24 \mathrm{kHz}$ (near one of the transducer's resonance frequencies) with a sine wave generating $\sim 500 \mathrm{~g}$ of surface acceleration for a total of 50 minutes using a function generator (33250A, Agilent Technologies) and driver/amplifier (PZD350A, Trek Inc.). Four advancing and receding CA measurements were taken at random locations and averaged for one coating data point. Measurements were taken before vibration and at intervals during vibration.

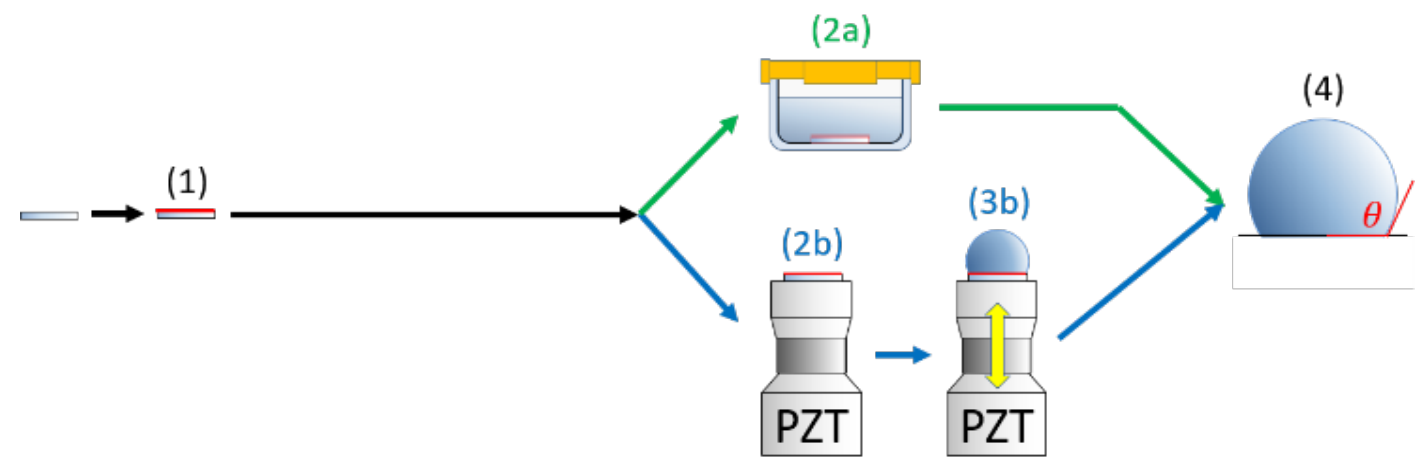

Figure 1: Flow-chart schematic of experimental testing. Glass microscope slides were coated with a hydrophobic coating (1). Coated glass slides were then either submerged in deionized water (2a), or fixed to a PZT (2b), then vibrated while covered with deionized water (3b). Advancing and receding contact angle was measured before, during, and after testing (4).

CA measurements were taken using the sliding angle (tilted plate) method. In this method, droplets were pumped on to the coating surface while the surface is oriented horizontally. The surface was tilted while the droplet shape was imaged. The advancing angle $\left(\theta_{A}\right)$ was taken as the angle at the leading edge of the droplet just before the leading edge of the droplet becomes unpinned, and the receding angle $\left(\theta_{R}\right)$ was taken similarly at the trailing edge of the droplet (Figure 2(b). The $\mathrm{CAH}$ was taken as the angle difference between the advancing and receding angle. Coated glass slides were fixed to a tilting goniometer to take CA measurements (Figure 2(a)). Deionized water droplets of $20 \mu \mathrm{L}$ were pumped onto the slides with a syringe pump (Legato 180, KD Scientific). A camera (NX8-S2, IDT Vision), and backlight were used to image droplets as the goniometer stage was tilted. Images of droplets were recorded at $30 \mathrm{~Hz}$. An edge detection algorithm $^{25-28}$ was used to track the CA at two sides of the droplet (Figure 2(c)).

While this measurement method is sufficient for characterization, it has its shortcomings. On a hydrophobic surface, the minimum $\mathrm{CA}$ is approximately equal to the receding $\mathrm{CA}$, while the maximum CA can be lower than the actual advancing CA. ${ }^{29}$ Drop placement also has an impact on sliding angle measurements due to surface heterogeneities and non-repeatable sliding behavior. Differing contact line lengths for a given droplet volume can cause for a range of equilibrium CAs on a surface. ${ }^{30}$ Despite these CAH measurement limitations, this method will suffice for detecting changes in $\mathrm{CAH}$ due to water exposure. 

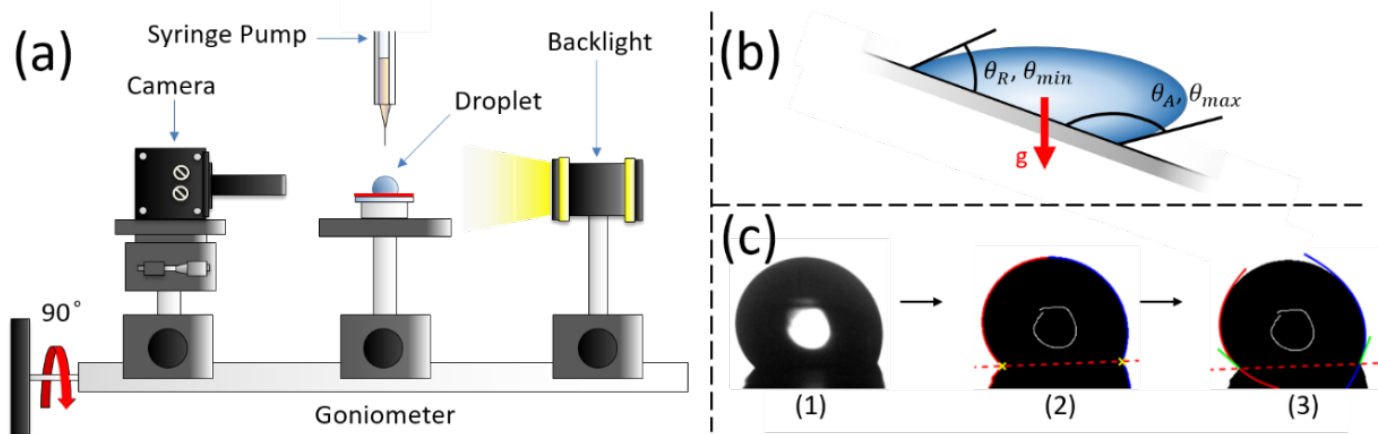

Figure 2: Experimental setup used to track droplet contact angle (a). Deionized water droplets were pumped onto hydrophobic-coated glass slides that were fixed to a tilting stage used to align imaging equipment.

Images were recorded at $30 \mathrm{fps}$ as the stage is tilted. Contact angle measurement diagram (b). Advancing $\left(\theta_{A}, \theta_{\text {max }}\right)$, and receding $\left(\theta_{R}, \theta_{\text {min }}\right)$ contact angles of a droplet the moment before it unpins and slides down a tilted surface. The direction of the gravitational force is represented with a red arrow for orientation purposes. MATLAB algorithm (c) used for tracking droplet position and contact angle. An image of a droplet (1) was separated into left and right sides and the contact line was plotted as a red dashed line through both yellow ' $x$ ' marks (2). Then, polynomial fitting of the droplet was used to find the contact angle of the droplet on each side (3).

\section{RESULTS AND DISCUSSION}

\section{SUBMERSION OF COATINGS}

Figure 3 shows advancing and receding CA data for coatings that were submerged in water as a function of time. All coatings but the FluoroSyl experienced degradation of their advancing and receding angles as a result of submersion in water. Both advancing and receding CAs degrade rapidly but moves toward a limiting value after 40 to 50 days of submersion in water.
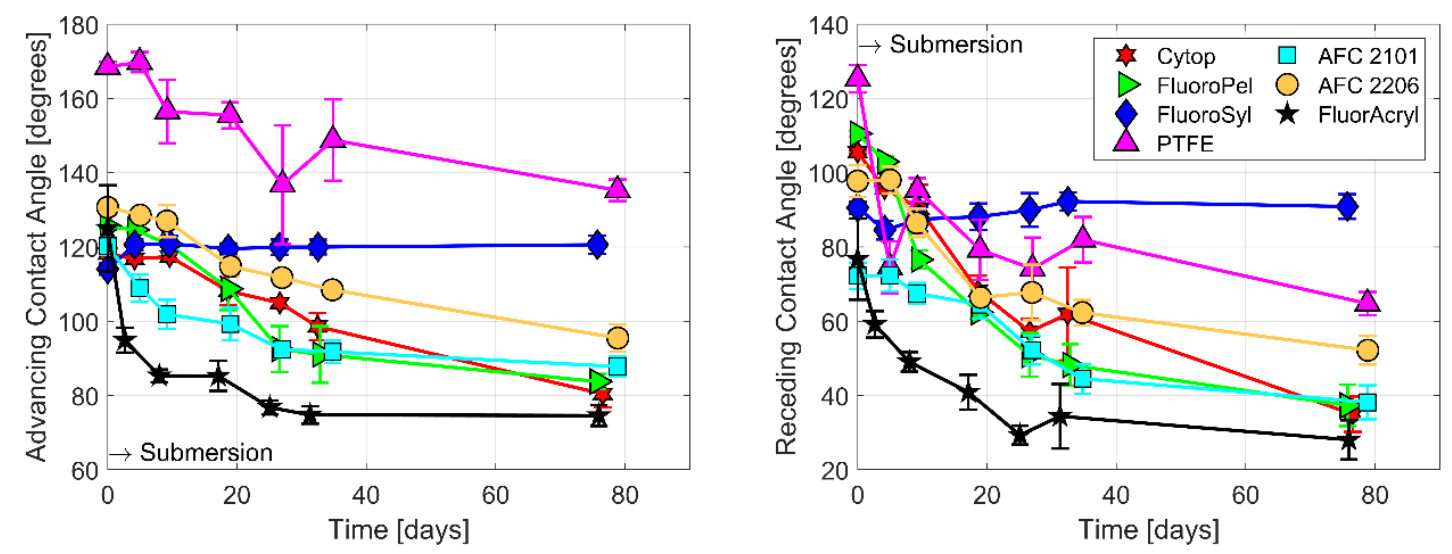

Figure 3: Advancing (left) and receding (right) contact angles for coatings that were submerged in water. Advancing and receding angles of coatings have decreased the longer the coating is submerged in water. Average contact angle measurements for each coating are plotted with straight lines connecting each data point as a visual guide. However, the advancing and receding angle of FluoroSyl does not follow this trend and appears to be resilient to submersion in water 
While decreasing advancing and receding angles was a common trend for 6 coatings, an increasing $\mathrm{CAH}$ for these coatings is not always manifest (Figure 4). An increase in CAH is only apparent for Cytop, FluoroPel, and AFC 2206 coatings. These three coatings experienced a more drastic decline of receding angle when compared to advancing angle, which in turn increases their CAH. Submersion of AFC 2101 and FluorAcryl results in similar degradation of advancing and receding $\mathrm{CA}$. Thus, a change in $\mathrm{CAH}$ is not apparent under the CA measurement uncertainty. Variation in CA measurements was particularly large for submerged PTFE results which could be a significant application issue itself.

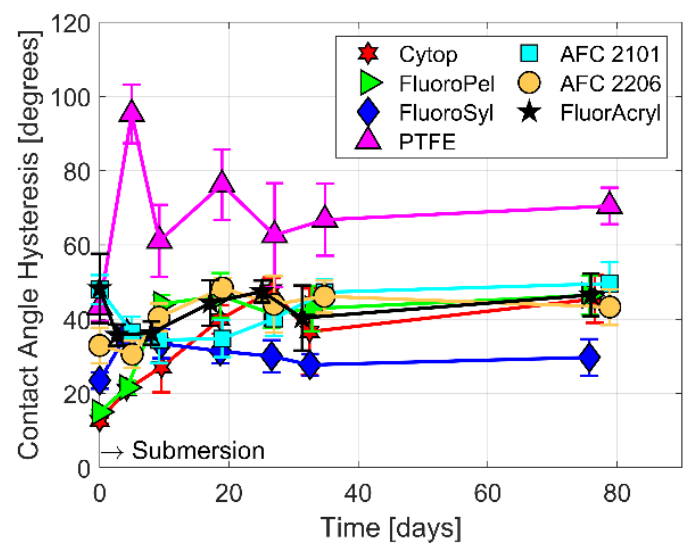

Figure 4: Change in contact angle hysteresis for coatings submerged in water determined by taking the difference in advancing and receding contact angles that were measured periodically. Average contact angle hysteresis measurements for each coating are plotted with straight lines connecting each data point as a visual guide. Cytop and FluoroPel coatings suffer an increase in contact angle hysteresis, while the trend of contact angle hysteresis for other coatings remains unclear, or within the bounds of error.

Because the exact composition of each proprietary coating is not known, it is difficult to hypothesize about the difference between the degradation trends of FluoroSyl and all other coatings tested. Boinovich et al. ${ }^{9}$ noted mechanisms (reversible and non-reversible) for the degradation of water CA on siloxane-based hydrophobic surfaces that were in continuous contact with water, including the growth of wetting films, reversible hydration creating hydrogen bonds, hydrolysis of molecule-specific free end groups with hydroxyl end groups, and non-reversible hydration of hydrogen bonding active groups inside the material. One possibility for the change in CA could be the absorption or adsorption of water during submersion. These effects could change surface energy, perhaps as a result of hydroxyl groups from water molecules changing the energy of the free surface by means of weak hydrogen bonding. Absorption might be evidenced by a change in film thickness as the coating is submerged in water. A contact profilometer (Dektak 150, Veeco) was used to measure the thickness of the Cytop coating. The initial Cytop coating thickness of $622 \mathrm{~nm}$ (6 $\mathrm{nm}$ standard deviation) increased to a thickness of $653 \mathrm{~nm}$ ( $30 \mathrm{~nm}$ standard deviation) after the coating was submerged for 12 days. The results of these measurements show that there may be a slight increase in thickness, but the measurement standard deviation is also increasing, so these results are insufficient to conclude that the change in wetting properties are due to the absorption of water. 


\section{HEAT TREATMENT OF SUBMERGED COATINGS}

It is possible for the coatings tested to absorb or adhere to hydroxyl groups of water molecules without causing a detectable film thickness change. Heating the submerged samples above $100^{\circ} \mathrm{C}$ could remove absorbed/adsorbed water and restore the initial wetting characteristics. Figure 5 shows the impact heating has on samples after the coatings were submersed in water for 130 days. The coatings were heat-treated in an oven at $160^{\circ} \mathrm{C}$, while CA measurements for each coating were taken periodically. The data suggests that the degradation of CA is at least partially thermally reversible for most coatings. This provides some evidence that coatings are absorbing or reacting with water, as heating coatings at $160^{\circ} \mathrm{C}$ seems to have a restoring effect. While the improvement may be due to evaporation of water from the coatings, the mechanism of the CA change is unclear. Heating produced significant CA recovery in all coatings tested in this study except for FluoroSyl, and FluorAcryl. Heat treatment of FluorAcryl and FluoroSyl may have been beneficial in restoring the CA if a lower temperature heating condition were chosen, however wetting characteristics of Cytop and PTFE were nearly completely restored as a result of heating at the given temperature.
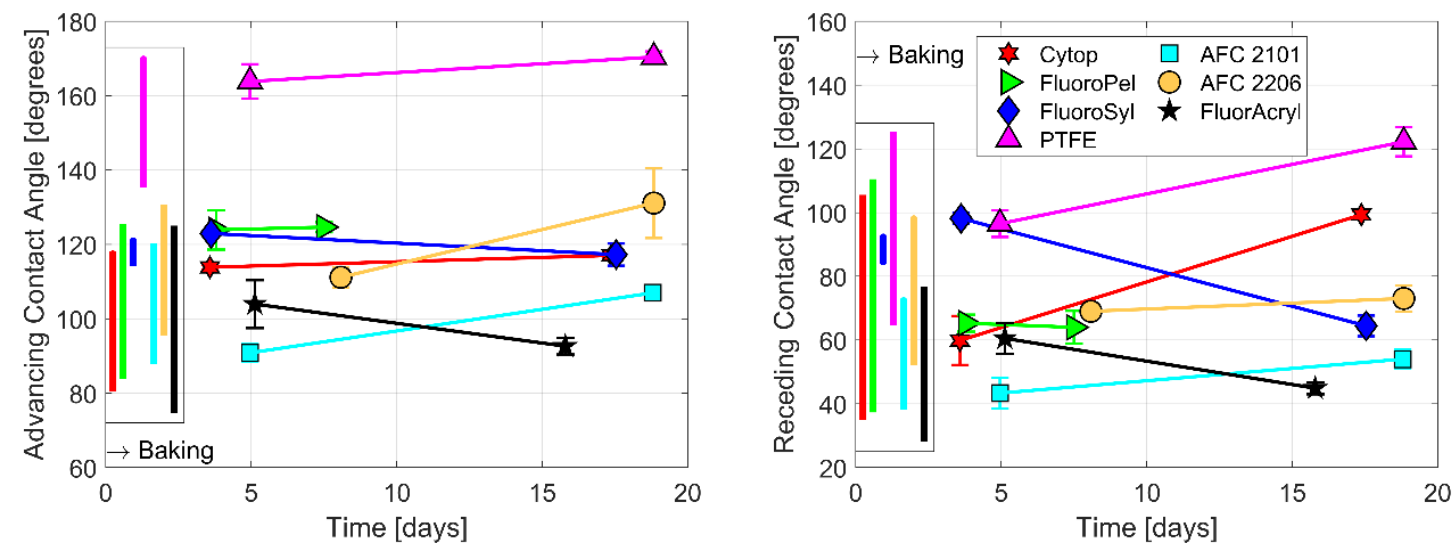

Figure 5: Change in advancing (left) and receding (right) contact angle by heating previously submerged samples at $160^{\circ} \mathrm{C}$ for varying lengths of time. Coatings were taken out of water after approximately 130 days to be subsequently heat-treated. Contact angles were measured periodically as coatings were heat-treated.

The box on the left side of each graph shows the range of contact angles for each coating as the coating degraded during submersion in water. This had a restorative effect for all coatings except FluoroSyl and Fluor Acryl.

The heat treatment rapidly restores the coatings compared to the rate at which they were degraded. While advancing and receding CA are improved individually, Figure 6 shows that the CAH for coatings except Cytop and PTFE are still increasing while undergoing heat treatment. AFC 2101, AFC 2206, and FluoroPel benefit from a restoration of advancing and receding angle as a result of heat treatment, however the rate of restoration for the receding angle lags the advancing angle, manifesting as an increase in $\mathrm{CAH}$ as heat treatment ensues. 


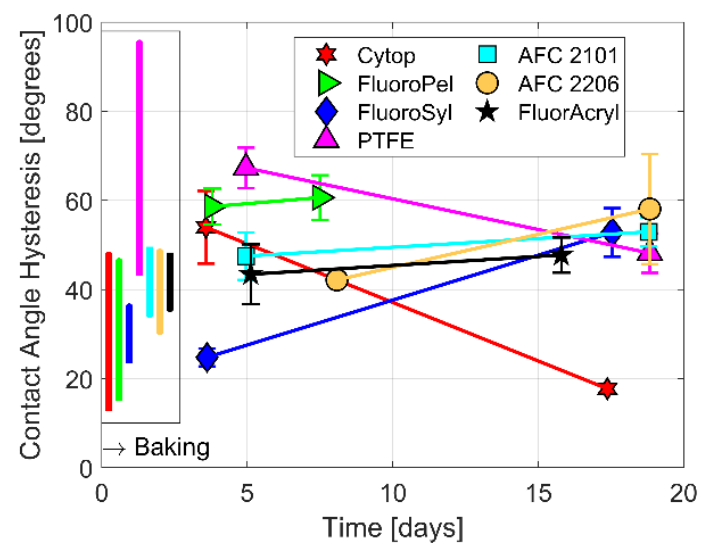

Figure 6: Change in contact angle hysteresis by heating previously submerged samples at $160^{\circ} \mathrm{C}$ for varying lengths of time. Coatings were taken out of water after approximately 130 days to be subsequently heattreated. Contact angle hysteresis for each coating was determined by taking the difference between the advancing and receding contact angles that were measured periodically as coatings were heat-treated. The box on the left side of the graph shows the range of contact angle hysteresis for each coating as the coating degraded during submersion in water. Heat treatment reduced the contact angle hysteresis for Cytop and PTFE coatings, but increased it for other coatings.

When FluorAcryl is heat-treated at $160^{\circ} \mathrm{C}$, the advancing and receding CA is initially restored very close to initial values, but as the heat treatment continues, the coating begins to degrade again. This coating yellowed when heat-treated, indicating some chemical/structural changes. Degradation of Fluorosyl's receding CA during heat treatment is attributed to exceeding the recommended $100^{\circ} \mathrm{C}$ curing temperature. However, this coating did not degrade due to water submersion, so heating to restore the coating is unnecessary.

One cause for CA change could be a change in surface topology, specifically roughness. Surfaces with micrometer and nanometer-scale roughness can exhibit hydrophobic and oleophobic properties due to contact line pinning and trapping of gas between the solid and liquid. ${ }^{31-33}$ If submersion of coatings in water results in a change in surface roughness, this could help explain the change in CA. Table 2 summarizes roughness data obtained with atomic force microscopy (DI3000, Digital Instruments) for both FluoroSyl and Cytop coatings before and after long-term submersion in water, and then again after being restored by heat treatment. The advancing (A) and receding $(\mathrm{R})$ contact angles for the coatings in each case are also referenced in the table. Cytop and FluoroSyl coatings were compared because of their contrasting reaction to testing.

Table 2: Average roughness, RMS roughness, and advancing (A) and receding (R) contact angle change for 2 coatings after water submersion and subsequent heat-treatment.

\begin{tabular}{|c|c|c|c|c|}
\hline & $\begin{array}{c}\text { Avg. } R_{a} \text { (Std. Dev.) } \\
{[\mathrm{nm}]}\end{array}$ & $\begin{array}{c}\text { Avg. } R_{q} \text { (Std. Dev.) } \\
{[\mathrm{nm}]}\end{array}$ & \multicolumn{2}{|c|}{$\begin{array}{c}\text { Contact Angle } \\
\text { [degrees] }\end{array}$} \\
\hline Cytop (As coated) & $0.5(0.0)$ & $0.7(0.0)$ & $\mathrm{A}: 118.2^{\circ}$ & $\mathrm{R}: 105.6^{\circ}$ \\
\hline Cytop (Submerged) & $19.8(2.1)$ & $42.8(4.7)$ & A: $80.4^{\circ}$ & $\mathrm{R}: 35.0^{\circ}$ \\
\hline Cytop (Heated $160^{\circ} \mathrm{C}$ ) & $10.7(5.3)$ & $15.3(7.3)$ & A: $117.1^{\circ}$ & $\mathrm{R}: 99.4^{\circ}$ \\
\hline FluoroSyl (As coated) & $15.4(11.9)$ & $40.0(29.9)$ & A: $114.1^{\circ}$ & $\mathrm{R}: 90.6^{\circ}$ \\
\hline FluoroSyl (Submerged) & $16.8(3.8)$ & $31.4(5.4)$ & A: $120.6^{\circ}$ & $\mathrm{R}: 90.9^{\circ}$ \\
\hline FluoroSyl (Heated $160^{\circ} \mathrm{C}$ ) & $2.7(0.8)$ & $6.8(1.6)$ & A: $117.3^{\circ}$ & $\mathrm{R}: 64.5^{\circ}$ \\
\hline
\end{tabular}


The average and RMS roughness of Cytop were both increased more than 10x due to submersion. The Cytop coating was only partially re-smoothed by heat treatment, but the advancing and receding CA were essentially restored in full. The roughness of FluoroSyl was not changed significantly due to submersion however, but remained close to the original roughness value. After heat-treating these two coatings at $160^{\circ} \mathrm{C}$, the average and RMS roughness were reduced. The CA characteristics of FluoroSyl were not affected by soaking the coating in water, however after heating the coating, the receding angle of water on FluoroSyl decreased. Roughness changes with heating are inconsistent with the CA changes between both materials, therefore the average roughness change that occurs as a result of submerging the coatings in water, and from heattreating the coatings at $160^{\circ} \mathrm{C}$, does not appear to be the primary cause of the CA changes.

Although the changing average and RMS roughness may not correlate well with CA change, the varying CA affects may still be related to changes on the surface of the coatings. Figure 7 shows atomic force micrographs of Cytop and FluoroSyl coatings that were taken immediately after the coatings were applied to the surface of a glass substrate. The spin-coated Cytop coating seems relatively flat and featureless compared to the uneven or splotchy surface features of the FluoroSyl coating that may be a result of non-uniform solvent evaporation during the coating curing process.

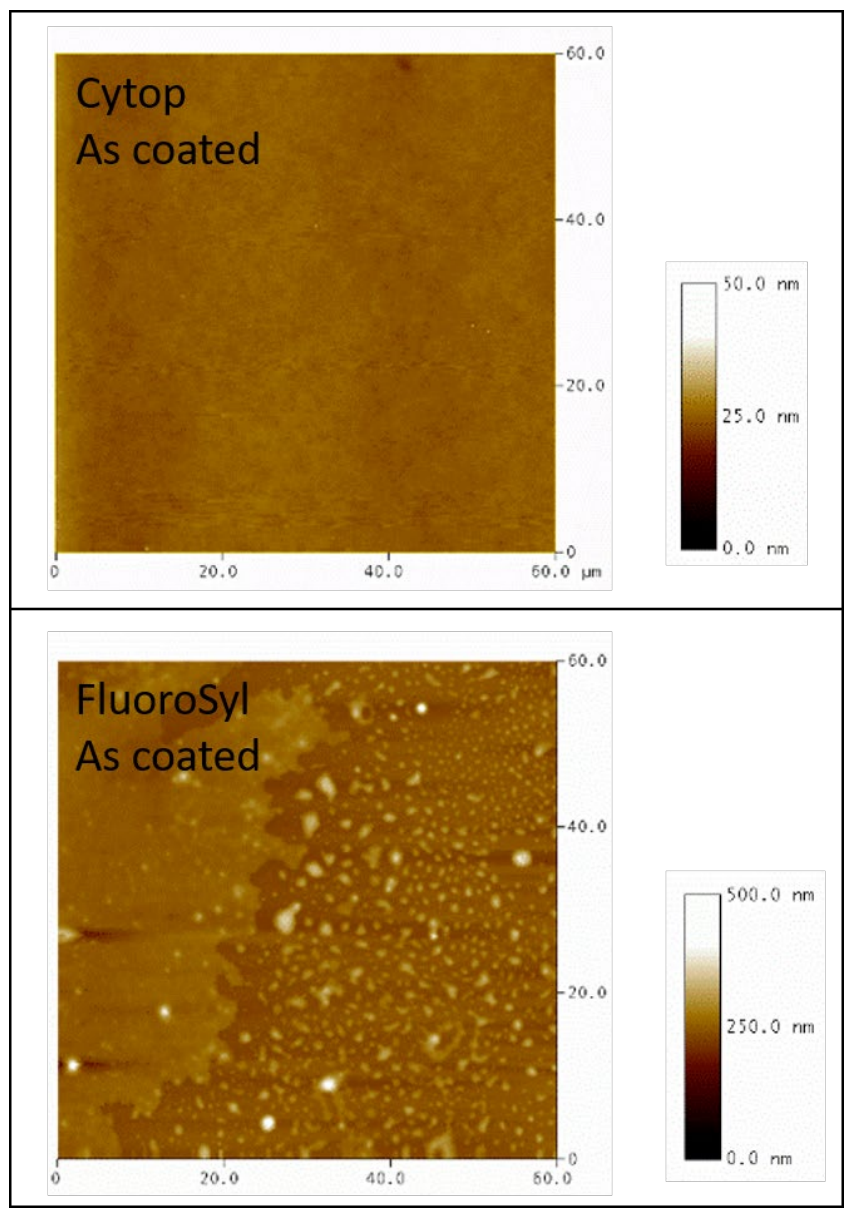

Figure 7: Atomic force micrographs of Cytop (top) and FluoroSyl (bottom) coatings immediately after the coatings were applied to the surface of a glass substrate. 
The uneven surface of the FluoroSyl coating could be the cause of the relatively large CAH due to composite wetting behavior along the different regions.

Atomic force micrographs of the surfaces before and after heating suggests that the response of the two coatings to the heat treatment is very different. Figure 8 shows atomic force micrographs of a single location on the surface of Cytop and FluoroSyl coatings after the coating was submerged in deionized water for $\sim 13$ months (Figure 8 left) and subsequently heat-treated for 4 days at $160^{\circ} \mathrm{C}$ (Figure 8 right). Long surface features resembling ridges appeared on both coatings after long periods of submersion in deionized water. These ridges could be indicative of coating buckling due to the absorption of water or due to increased swelling at the free edges of a crack. While these ridges appear in both coatings, it does not explain the change in CA as the FluoroSyl coating had negligible change in CA as a result of submersion.

The ridges that appeared on both coatings after long periods of submersion were reduced in size in both instances after heat treatment at $160^{\circ} \mathrm{C}$ for 4 days. The surface of the Cytop coating began to warp or become wave-like, but the small surface features were smoothed after the submerged sample was heat-treated. The smoothing of the Cytop coating as a result of heat treatment could be responsible in the restoration of the water CA. The water CA of the FluoroSyl coating, on the other hand, was degraded as a result of the same heat treatment. The ridges that appeared on the surface of FluoroSyl after submersion were reduced in size, however, the splotchy surface features were removed and replaced by many small features that look like tiny droplets resting all over the surface. These tiny features may be indicate coating de-wetting as a result of exposure to a temperature above the glass transition temperature. The formation of the tiny features on the surface of FluoroSyl after the coating was heat-treated are most likely the cause for the degradation of the water CA that was observed.

It is clear that some morphology changes may be playing a role in the changing CA of hydrophobic coatings. However, it is also likely that some chemical changes are taking place. The buckling and formation of additional surface features that appear when coatings are submerged would act to pin the contact line of droplets wetting the surface thereby increasing the advancing and decreasing receding CAs. Additionally, the possible cracking or de-wetting of coating surfaces could be exposing hydrophilic glass surfaces that could act like barriers to contact line motion. This effect would account for the degradation of the receding angle of droplets wetting the surface, as well as increase the $\mathrm{CAH}$. 


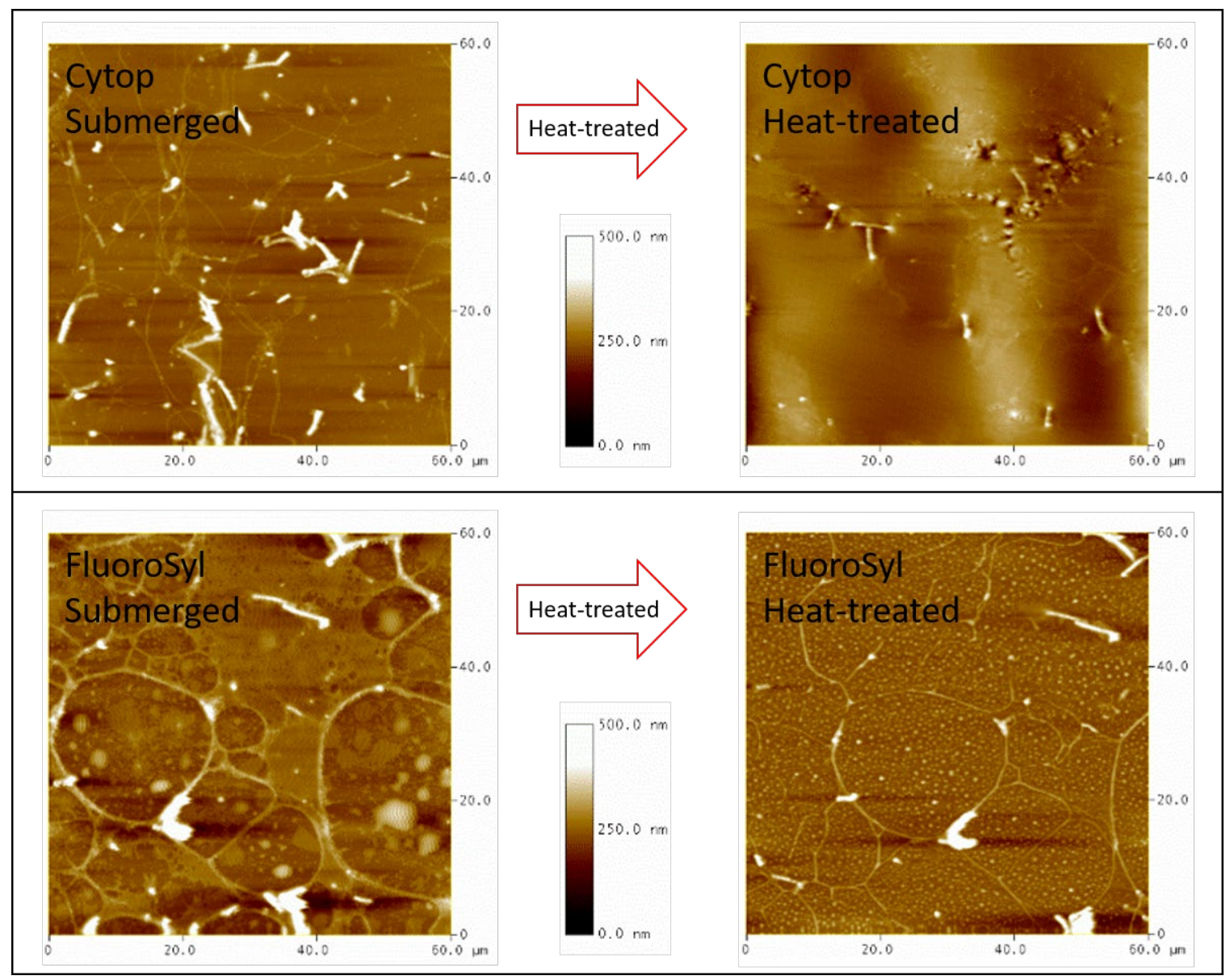

Figure 8: Atomic force micrographs of a single location on the surface of Cytop (top) and FluoroSyl (bottom) coatings on a glass substrate after the coating was submerged in deionized water for $\sim 13$ months (left) and subsequently heat-treated for 4 days at $160^{\circ} \mathrm{C}$ (right).

Water submersion provides valuable insight into the performance of the materials during water exposure, but the tests are time consuming. Methods of accelerated testing are of great value in shortening testing times and predicting long term coating performance. While temperature is widely used in accelerated degradation testing, it can cause unexpected changes such as a phase change. Alternatively, acceleration of coating degradation from additional motion of submerged coatings could occur as a result of increased transport between water and substrate.

\section{COMPARISON OF ULTRASONICALLY VIBRATED WET COATINGS}

Ultrasonic vibration of coated substrates covered with water is capable of altering the hydrophobic properties of fluoropolymer coatings as seen in Figure 9. In general the changes are less drastic, but this may just be due to the shorter duration of degradation when compared to long term submersion. 

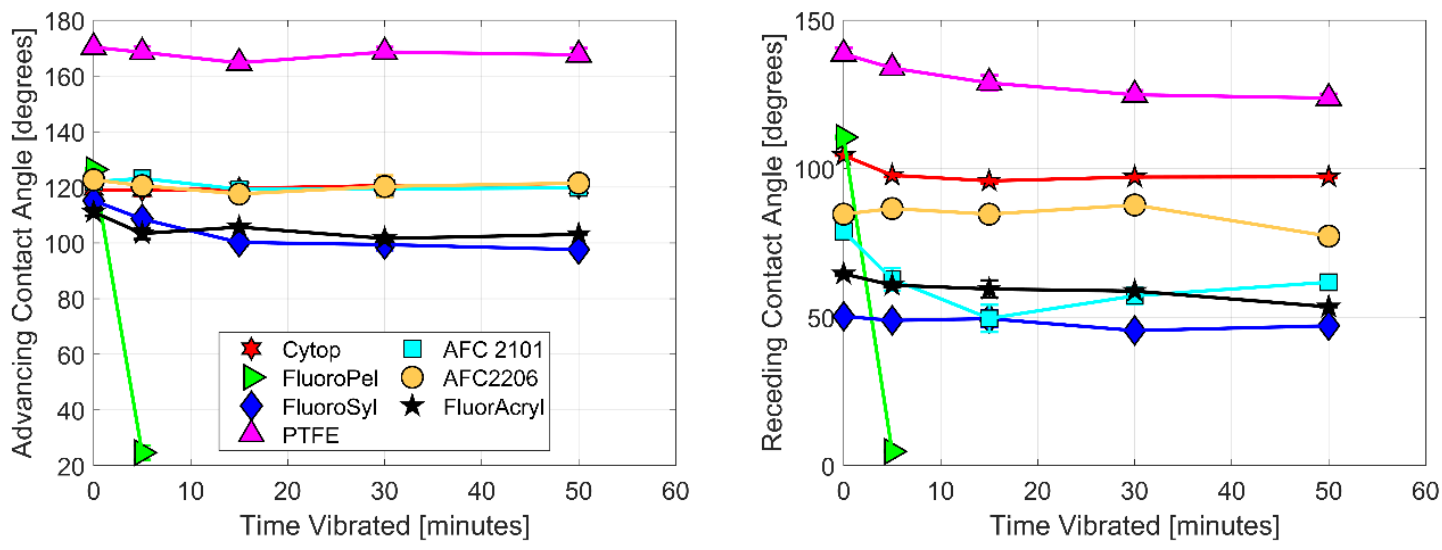

Figure 9: Advancing (left) and receding (right) contact angle for coatings that were covered in water and vibrated with a piezoelectric transducer (sine wave, $24 \mathrm{kHz}, \sim 500 \mathrm{~g}$ maximum surface acceleration). Average contact angle measurements for each coating are plotted with straight lines connecting each data point as a visual guide. Vibration caused slight degradation for most coatings except for AFC 2206 and Cytop, and severe degradation for FluoroPel.

Vibrating of coatings while covered in water produced varying results in comparison to soaking tests. Most coatings showed negligible degradation from water-covered vibration during the duration of testing, however FluoroPel degraded quickly compared to all other coatings. While the degradation of some coatings is apparent, some coatings like Cytop have negligible change in the amount of time vibrated, but did degrade significantly when submerged in water. It is apparent that ultrasonic vibration increases the degradation rate of coatings that degraded substantially in the time vibrated, but the degradation of each coating in one method are not proportional to the other method over the range of conditions studied. Figure 10 compares the final measured advancing and receding CA for each method of degradation. Distinctive degradation of FluoroPel and FluoroSyl by vibration demonstrate that vibration does not simply accelerate the changes observed from water immersion as only one of these coatings was affected by submersion. This data, like the data in Figure 3, also shows that the receding CA degrades more than the advancing CA. Surface acceleration levels and rate/amount of coating CA degradation were not apparently related when comparing samples that were vibrated between 700 and 7000 g. FluoroPel degraded most substantially and at the highest rate due to ultrasonic agitation while covered in water. While the advancing and receding CAs for FluoroPel were restored significantly by heat treatment after submersion, this coating was not restorable after undergoing ultrasonic vibration while covered with water. Follow-up profilometry testing revealed that FluoroPel was removed in the regions covered by water when vibrated. 

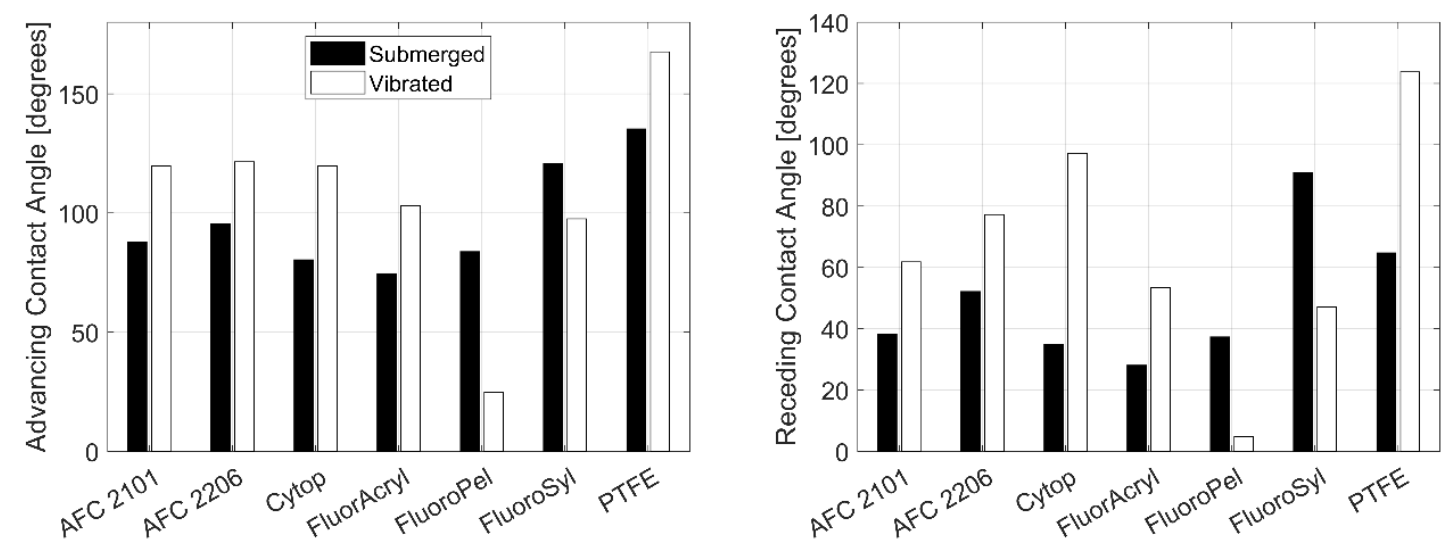

Figure 10: Final measured advancing (left) and receding (right) contact angle of coatings that were tested by both means of degradation. Distinctive degradation of FluoroSyl and FluoroPel suggests that the degradation methods are not very similar.

\section{CONCLUSION}

Degradation of seven hydrophobic fluoropolymer coatings were tested with two different methods: submersion of the coating in deionized water over time, and ultrasonic vibration of the coating while covered in deionized water. These two scenarios represent real conditions that a hydrophobic coating may be exposed to in various applications. While FluoroSyl experienced negligible CA change while submerged in water, the CA in all other coatings tested was reduced by $20-70 \%$. Heat treatment was shown to restore the CA of submerged coatings over a shorter time scale - as compared to degradation during submersion - when coatings were not sensitive to high temperatures, and careful selection of heating conditions were chosen. Submersion of coatings in water results in a slow degradation of CA occurring over the course of days to weeks, while vibration of most coatings showed some modest degrading effects from a short exposure (minutes instead of days) to vibration while covered in water. High frequency vibration of wet coatings is not simply an acceleration of the degradation that occurs as a result of submerging the coatings in water - as distinct trends are apparent for FluoroPel and FluoroSyl - but it could be useful as an accelerated indication of how hydrophobic coatings subjected to vibration or agitation in industrial applications will degrade. Surface profilometry and atomic force microscopy measurements did not reveal a recognizable trend in surface characteristics (coating swelling and surface roughness) that would completely explain the changing CA. However, atomic force microscopy did reveal both similar and differing surface changes on different coatings that help to explain some of the CA changes on the various coatings. The degrading behavior of hydrophobic coatings exposed to water should be considered on a coating-to-coating basis as different coatings react differently. It does not appear that vibration of coatings submerged in water is promising as an expedited method to determine if and how coatings will degrade due to water exposure or submersion over the lifetime of the coating as both methods of degradation are inconsistent between the coatings tested.

\section{ACKNOWLEDGMENTS}

This work was supported by the National Science Foundation under Grant No. 1361919. 


\section{REFERENCES}

1. R. Asmatulu, G. A. Mahmud, C. Hille, and H. E. Misak, "Effects of UV degradation on surface hydrophobicity, crack, and thickness of MWCNT-based nanocomposite coatings," Progress in Organic Coatings, vol. 72, pp. 553-561, 2011/11/01/2011.

2. X. F. Yang, D. E. Tallman, G. P. Bierwagen, S. G. Croll, and S. Rohlik, "Blistering and degradation of polyurethane coatings under different accelerated weathering tests," Polymer Degradation and Stability, vol. 77, pp. 103-109, 2002/01/01/2002.

3. V. Gutiérrez-Cano, F. Rodríguez, J. A. González, and V. Díaz, "Thermal degradation of hydrophobic graphite-based thin film nano-coatings observed by Raman spectroscopy," Thin Solid Films, vol. 648, pp. 8-11, 2018/02/28/ 2018.

4. Y. Han Yeong, A. Steele, E. Loth, I. Bayer, G. De Combarieu, and C. Lakeman, "Temperature and humidity effects on superhydrophobicity of nanocomposite coatings," Applied Physics Letters, vol. 100, p. 053112, 2012/01/30 2012.

5. S. A. Mahadik, P. D. Fernando, N. D. Hegade, P. B. Wagh, and S. C. Gupta, "Durability and restoring of superhydrophobic properties in silica-based coatings," Journal of Colloid and Interface Science, vol. 405, pp. 262-268, 2013/09/01/ 2013.

6. W.-G. Ji, J.-M. Hu, J.-Q. Zhang, and C.-N. Cao, "Reducing the water absorption in epoxy coatings by silane monomer incorporation," Corrosion Science, vol. 48, pp. 3731-3739, 2006/11/01/ 2006.

7. C. Vosgien Lacombre, G. Bouvet, D. Trinh, S. Mallarino, and S. Touzain, "Water uptake in free films and coatings using the Brasher and Kingsbury equation: a possible explanation of the different values obtained by electrochemical Impedance spectroscopy and gravimetry," Electrochimica Acta, vol. 231, pp. 162-170, 2017/03/20/ 2017.

8. Q. Zhou and Y. Wang, "Comparisons of clear coating degradation in $\mathrm{NaCl}$ solution and pure water," Progress in Organic Coatings, vol. 76, pp. 1674-1682, 2013/11/01/ 2013.

9. L. Boinovich, A. M. Emelyanenko, and A. S. Pashinin, "Analysis of Long-Term Durability of Superhydrophobic Properties under Continuous Contact with Water," ACS Applied Materials \& Interfaces, vol. 2, pp. 1754-1758, 2010/06/23 2010.

10. Q. Zhou, Y. Wang, and G. P. Bierwagen, "Flow accelerated degradation of organic clear coat: The effect of fluid shear," Electrochimica Acta, vol. 142, pp. 25-33, 2014/10/01/ 2014.

11. Z. Yuan, X. Wang, J. Bin, C. Peng, S. Xing, M. Wang, et al., "A novel fabrication of a superhydrophobic surface with highly similar hierarchical structure of the lotus leaf on a copper sheet," Applied Surface Science, vol. 285, pp. 205-210, 2013/11/15/2013. 
12. J. P. Youngblood, L. Andruzzi, C. K. Ober, A. Hexemer, E. J. Kramer, J. A. Callow, et al., "Coatings Based on Side-chain Ether-linked Poly(ethylene glycol) and Fluorocarbon Polymers for the Control of Marine Biofouling," Biofouling, vol. 19, pp. 91-98, 2003/01/01 2003.

13. A. V. Rao, S. S. Latthe, S. A. Mahadik, and C. Kappenstein, "Mechanically stable and corrosion resistant superhydrophobic sol-gel coatings on copper substrate," Applied Surface Science, vol. 257, pp. 5772-5776, 2011/04/15/ 2011.

14. Y. Liu, Z. Lin, K. Moon, and C. P. Wong, "Superhydrophobic Nanocomposite Coating for Reliability Improvement of Microelectronics," IEEE Transactions on Components, Packaging and Manufacturing Technology, vol. 3, pp. 1079-1083, 2013.

15. P. Arunkumar, Y. H. Kim, H. J. Kim, S. Unithrattil, and W. B. Im, "Hydrophobic Organic Skin as a Protective Shield for Moisture-Sensitive Phosphor-Based Optoelectronic Devices," ACS Applied Materials \& Interfaces, vol. 9, pp. 7232-7240, 2017/03/01 2017.

16. H. W. Lu, K. Glasner, A. L. Bertozzi, and C. J. Kim, "A diffuse-interface model for electrowetting drops in a Hele-Shaw cell," Journal of Fluid Mechanics, vol. 590, pp. 411$435,2007$.

17. K. Bonhye and K. Chang-Jin, "Evaluation of repeated electrowetting on three different fluoropolymer top coatings," Journal of Micromechanics and Microengineering, vol. 23, p. 067002, 2013.

18. V. Arcella, A. Ghielmi, and G. Tommasi, "High Performance Perfluoropolymer Films and Membranes," Annals of the New York Academy of Sciences, vol. 984, pp. 226-244, 2006.

19. Cytop Catalog. Available: http://www.bellexinternational.com/products/cytop/pdf/cytopcatalog.pdf

20. S. Simal, J. Benedito, E. S. Sánchez, and C. Rosselló, "Use of ultrasound to increase mass transport rates during osmotic dehydration," Journal of Food Engineering, vol. 36, pp. 323$336,1998 / 05 / 01 / 1998$.

21. Bellex, "Cytop Catalog," ed, 2009.

22. Cytonix. FluoroPel Coatings Selection Guide. Available: https://www.cytonix.com/Articles.asp?ID=252

23. Cytonix. FluorAcryl 3298 Datasheet. Available: https://www.cytonix.com/Articles.asp?ID=277

24. Cytonix. FluoroSyl 3750 Datasheet. Available: https://www.cytonix.com/FluoroSyl-3750p/fsm3750 60ml.htm 
25. A. Trujillo-Pino, K. Krissian, M. Alemán-Flores, and D. Santana-Cedrés, "Accurate subpixel edge location based on partial area effect," Image and Vision Computing, vol. 31, pp. 72-90, 2013/01/01/2013.

26. A. Trujillo-Pino, "Accurate subpixel edge location," ed, 2017, p. Detection of subpixel edges with very high precision in grey level images.

27. O. Gal, "fit_ellipse," ed, 2003, p. Find the best fit for an ellipse using a given set of points (a closed contour).

28. N. K. Andersen, "Drop Shape Analysis," ed, 2016, p. Fit contact angle by double ellipses or polynomials.

29. B. Krasovitski and A. Marmur, "Drops Down the Hill: Theoretical Study of Limiting Contact Angles and the Hysteresis Range on a Tilted Plate," Langmuir, vol. 21, pp. 3881$3885,2005 / 04 / 012005$.

30. E. Pierce, F. J. Carmona, and A. Amirfazli, "Understanding of sliding and contact angle results in tilted plate experiments," Colloids and Surfaces A: Physicochemical and Engineering Aspects, vol. 323, pp. 73-82, 2008/06/20/ 2008.

31. D. Ebert and B. Bhushan, "Durable Lotus-effect surfaces with hierarchical structure using micro- and nanosized hydrophobic silica particles," Journal of Colloid and Interface Science, vol. 368, pp. 584-591, 2012/02/15/2012.

32. L. Feng, S. Li, Y. Li, H. Li, L. Zhang, J. Zhai, et al., "Super-Hydrophobic Surfaces: From Natural to Artificial," Advanced Materials, vol. 14, pp. 1857-1860, 2002.

33. T. Hang, A. Hu, H. Ling, M. Li, and D. Mao, "Super-hydrophobic nickel films with micronano hierarchical structure prepared by electrodeposition," Applied Surface Science, vol. 256, pp. 2400-2404, 2010/02/01/ 2010. 